\title{
PREDICTING SPEECH INTELLIGIBILITY FOR HEARING AID USERS IN THE PRESENCE OF DIGITAL WIRELESS PHONE INTERFERENCE
}

\author{
Tamy L. Fry, Robert E. Schlegel, Randa L. Shehab, and Hank Grant \\ School of Industrial Engineering \\ University of Oklahoma \\ Norman, OK 73019
}

\section{INTRODUCTION}

By mid-March of 1999 there were more than 69.4 million wireless phone subscribers in the United States. More than 11 million of these subscribers used digital phones, which transmit a higher quality voice signal to the user but have the potential to interact negatively with sensitive electronic devices. For example, acoustic interference may occur in hearing aids when the hearing aid amplifier demodulates the RF transmission signal produced by a digital wireless phone. This noise can resemble static hiss, clicking, or buzzing, depending on the particular phone technology. Although not biologically harmful, the interference is annoying and can seriously degrade the intelligibility of the speech message.

\section{CLINICAL STUDY I}

Clinical data from sixty-one hearing-impaired participants were evaluated to investigate the impact of hearing loss configuration (flat, sloping, ski slope, and rising), hearing loss severity (normal, mild, moderate, moderately severe, severe, and profound), digital phone technology (TDMA-50 Hz at $800 \mathrm{MHz}, \mathrm{CDMA}$ at $800 \mathrm{MHz}$, and TDMA-217 $\mathrm{Hz}$ at $1900 \mathrm{MHz}$ ), and hearing aid type (BTE, ITE, ITC, and CIC) on the speech intelligibility of hearing aid wearers in the presence of wireless phone interference. The analyses confirmed that phone technology and hearing loss severity were significant factors in the determination of the speech intelligibility scores. As expected, the highest intelligibility was found in the "No RF" condition. Significant decreases in average speech intelligibility were found with CDMA, TDMA-217 $\mathrm{Hz}$, and TDMA-50 Hz phone technologies, respectively. In general, hearing loss configuration and hearing aid type were not found to have a statistically significant effect on speech intelligibility.

A previously unreported relationship between speech intelligibility, phone technology, and hearing loss severity was established. Speech intelligibility was found to decrease proportionally with increasing hearing loss severity, independent of phone technology. For each $1 \mathrm{~dB}$ increase in hearing loss severity, speech intelligibility decreased by one percent.

\section{CLINICAL STUDY II}

A second study evaluated the accuracy of the Speech Intelligibility Index (SII), a revised version of the Articulation Index (AI), in predicting speech intelligibility for hearing-impaired listeners in the presence of wireless phone interference. The complex SII methodology permits an adjustment of the input signal for acoustic gain or loss. Hence, this method may be more applicable than the AI for hearing aid wearers. However, the standard has neither been validated nor recommended for hearing-impaired listeners.

Speech intelligibility scores were obtained from twenty-four hearing-impaired participants who were presented with $65 \mathrm{~dB}$ speech mixed with recorded interference from each of the three wireless phone technologies at two sound pressure levels ( 35 and $65 \mathrm{~dB}$ SPL). The correlation of the respective SII predicted values with the measured speech intelligibility scores $(\mathrm{r}=0.970)$ was very similar to that for the AI $(r=0.965)$. Unfortunately, the SII was no more accurate than the $\mathrm{AI}$ in predicting the speech intelligibility for hearing aid wearers in the presence of wireless phone interference. Considering the input requirements and the difficulty of the calculations, the SII was judged to be a less effective and less efficient method for accurately predicting the speech intelligibility. 